\title{
Multilevel Image based Data Security using Combined Approach of Lsb_Dct Watermarking
}

\author{
Ranjeet Kumar Singh \\ M.Tech Department of I.T \\ Govt.Engg.College, Ajmer
}

\author{
Deepak Gupta \\ Asst. Prof. Department of CS \\ Govt. Engg. College, Ajmer
}

\author{
Santosh Kumar Singh \\ M.Tech Department of I.T \\ Govt.Engg.College, Ajmer
}

\begin{abstract}
Digital watermarking is a method which is used to verify texts, image, video etc. Watermarking Technique is not only used for Authentication, it's also used for security, image protection, rights of documents. In this perspective experiment we used edge detection technique in watermarking for the purpose of highly authentication of image. Here we used combined approach of LSB-DCT watermarking for multilevel image based data security, in LSB-DCT approach author used edge of original image as a watermark image which is calculated by sobel edge detection technique this watermark is embedded on original image with the help of LSB watermark approach. After the first watermarking process is completed author embedded next watermark on the original image with the help of DCT Watermarking. In this experiments author explain effect of noise on dual watermarked image with the help of PSNR, SNR and Correlation coefficient.
\end{abstract}

\section{Keywords}

LSB, DCT Watermarking, Edge Detection, PSNR, SNR.

\section{INTRODUCTION}

Digital watermarking is a method which allows a separate to enhance secreted copyright announcements or additional confirmation messages to digital image, audio, or video signals and documents. Image watermarking provide security of paper currency and other important .Digital watermarking can be a form of steganography [1], in which data is hidden in the message without the end user's awareness.Digital watermarking is a process of inserting data (watermark) into a hypermedia object to help to defend the owner's right to that object. The inserted data (watermark) may be either visible or invisible [5].

\section{LSB WATERMARKING:}

Now a digital image, data can be inserted directly into each bit of image data or the more busy areas of an image can be calculated so as to hide such Messages in less noticeable parts of an image. Tirkel et al were one of the first used methods for image watermarking. Two techniques were offered to hide facts in the spatial domain of images by them. These approaches were constructed on the pixel value's Least Significant Bit (LSB) adjustments. The algorithm offered by Kurah and McHugh's to insert in the LSB and it was known as image downgrading [7].

\section{DCT WATERMARKING:}

Discrete-Cosine-Transform or DCT is a standard transform domain Watermarking technique. The DCT permits an image to be fragmented up into different frequency bands i.e. the high, middle and low frequency bands so creation it at ease to choose the band in which the watermark is to be inserted. The literature survey tells that generally the middle frequency bands are chosen because inserting the watermark in a middle frequency band does not scatter the watermark info to greatest visual significant parts of the image i.e. the low frequencies and also it do not overexpose them to deletion through compression and noise attacks where high frequency components are targeted [8].

\section{EDGE DETECTION}

Edge detection is the method of localizing pixel intensity changes. The edge detection has been used by several areas such as segmentation, object recognition, and target tracking, etc. Therefore, the edge detection is main parts of image processing. There mostly exist several edge detection approaches (Sobel [9, 10], Prewitt [11], Roberts [12], and Canny [13]). These methods have been offered for identifying changes in images. Early methods determined the best gradient operator to detect sharp intensity variations [14]. Normally apply derivative operation on image for identifying edge. Derivative based approaches can be characterized into two sets, specifically first and second order derivative approaches. First order derivative based methods depend on calculating the gradient some directions and merging the result of each gradient. The value of the gradient magnitude and orientation is estimated using two differentiation masks [15].

\section{ALGORITHM STEPS:}

1) Take an original image.

2) Edge Detection of Original Image Using Sobel Edge Detection Operator.

3) Consider the edged of original image from step2 as watermark1 image.

4) Replace LSB of Original Image by MSB of watermark1 image (edge of original image).

5) Display original image and watermarked1 image.

6) Consider Watermarked1 image as original image for DCT Watermarking

7) Load any other new image as a watermark.

8) Apply DCT Watermarking, to find watermarked 2 image.

9) Apply inverse DCT we find watermark2 and original image (watermarked1 image).

10) Apply Inverse of LSB to find watermark1 (edge of original image) and original image. 


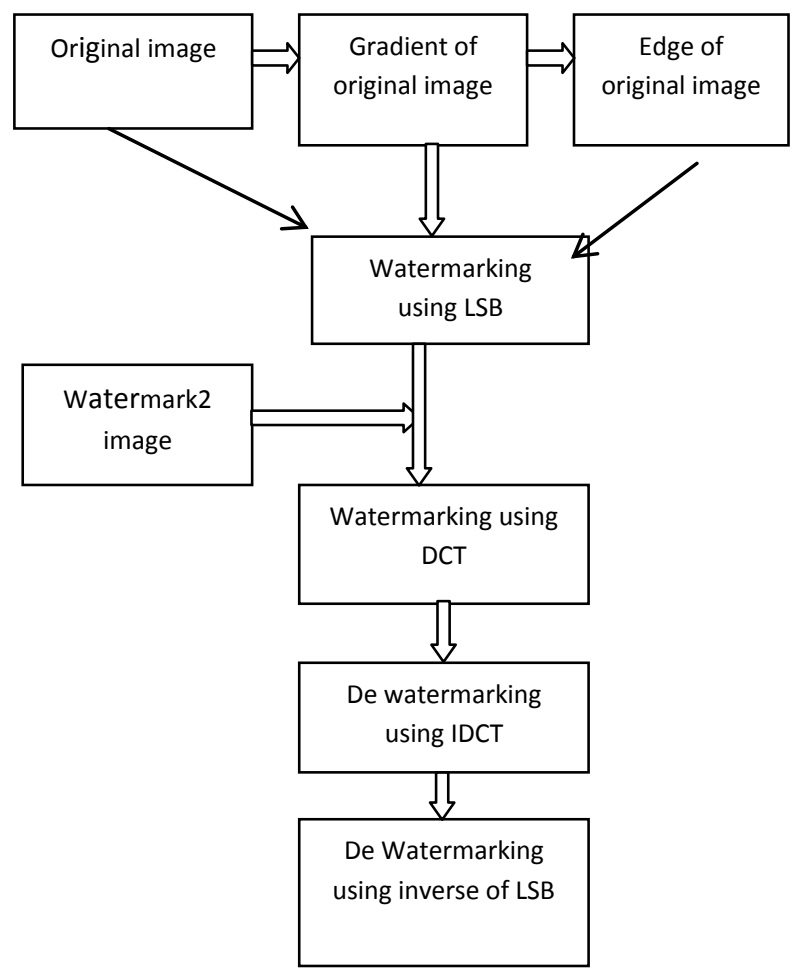

Key part of this paper is effect of noise on dual watermarked image. In figure 2, this describes the impact of noise (different-2 type of noise) on watermarked image. Its explain what the effect of noise with different-different variance on the watermarked image. Firstly we see the watermark1 is embedding in original image using LSB Watermarking process after LSB approach watermark2 is inserting in watermarked1 image using DCT approach. After the completion of dual watermarking process author explain impact of noise on dual watermarked image. In figure 2 it shows the attack of different- 2 type of noise on thedual watermarked image. in this paper author simulate the experimental result using MATLAB software here we simulate what the effect of watermark and recover watermark image when the noise are attacked on watermarked image.

In the below Table.1 it show the PSNR (peak signal to noise ratio), MSE (mean squared error), and Histogram of images. Here we see the PSNR between watermarked 1 and watermarked 2 image is high compare to other but MSE is low. And similarly PSNR between watermark2 and recoverwatermark2 is low but MSE is high compare to other.

Figur1. Dual watermarking using LSB_DCT Approach

Table1: histogram, psnr, mse comparisons of image

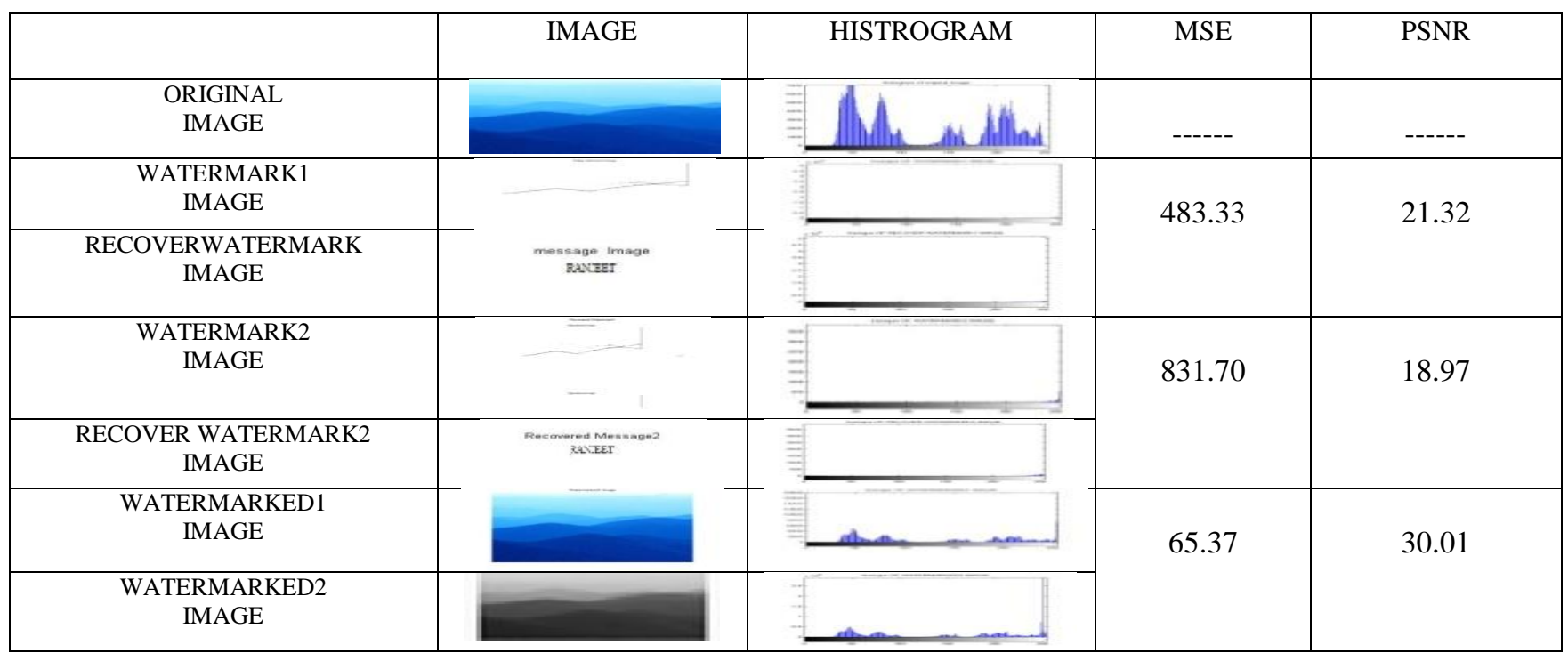




\section{EFFECT OF NOISE}

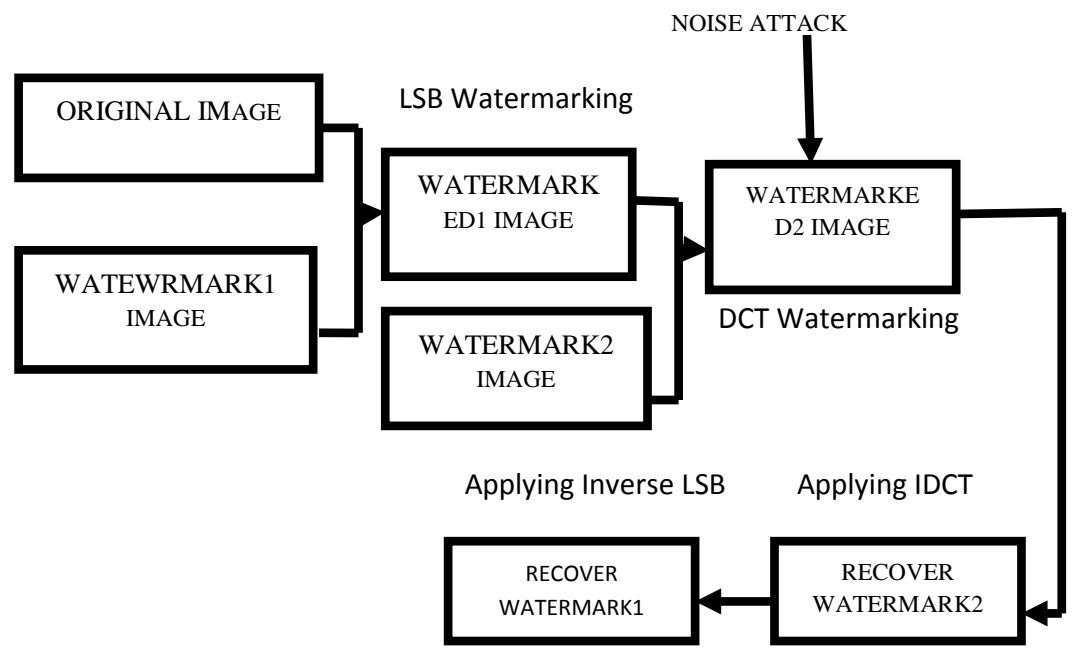

Figure2: attack of noise on dual watermarked image

For checking the performance of above algorithm, the experiments are simulated with the MATLAB software. The experiments follow the conversion of original image into edge of original image using sobel operator, this edge image works as watermark1 image and it embed into original image using LSB watermarking and find watermarked1 image. After the LSB watermarking author take another watermark image (watermark2) which embed with watermarked1 image with the help of DCT watermarking and obtained watermarked2 image after the competition of watermarking process we used de-watermarking process one by one. At first we used IDCT process and find recover image (watermark2 image), after IDCT author used Inverse of LSB and find recover image (watermark1 image).

\section{EXPIREMENTAL RESULT AND}

\section{ANALYSIS:}

In this experiment author mainly show the effect of noise on watermarked image. In table2, table 3 and table4 represent PSNR, SNR, and CORRELATION COEFFICIENT between watermark image (watermark1 and watermark2) and recover watermark image and explain what the effect of quality of image when different-2 noise are attacked on watermarked image table2 represent quality of image in the form of SNR (signal to noise ratio), PSNR (peak signal to noise ratio), CC (correlation coefficient).table 3 show the effect of speckle noise with different-2 variance on dual watermarked image Similarly table4 show the effect of salt and pepper noise with different-2 variance on dual watermarked image and table4 explain the impact of Gaussian noise with different-2 variance on dual watermarked image. In table2, table 3 and table4, W1 Represent watermark1 image, RW1 Represent recover watermark1 image after the completion of watermarking process .similarly W2 Represent Watermark2 image and RW2 Represent recover watermark2 image.

In the below Graph1 [A], Graph1 [B], Graph1[C] and Graph2 [A], Graph2 [B] Show the image quality when different-2 noise are attacked on watermarked image. In graph1[A],Graph1[B],Graph[C] represent the PSNR values between watermark1 and recover watermark1 is more when the salt $\&$ pepper noise are attacked compare to speckle and
Gaussian noise. Similarly SNR values are also high between them compare to speckle and Gaussian noise. But Correlation Coefficient is high when speckle noise is attacked.

Similarly In Graph2[A],Graph2[B] represent the PSNR values between watermark 2 and recover watermark 2 is more when the salt $\&$ pepper noise are attacked compare to speckle and Gaussian noise. Similarly in Graph2 [B] show SNR values are also high between them compare to speckle and Gaussian noise.

Table2: Impact of Speckle noise on watermark and Recover watermark

\begin{tabular}{|c|c|c|c|}
\hline \multirow{2}{*}{ COMPARISION } & W1 \& RW1 & W2 \& RW2 \\
\hline \multirow{2}{*}{$\begin{array}{c}\text { PSNR, SNR AND } \\
\text { CORRELATION } \\
\text { COEFFICIENT WHEN } \\
\text { NOISE VARIANCE IS 0.020 }\end{array}$} & PSNR & 14.6150 & 15.9095 \\
\cline { 2 - 4 } & SNR & 9.8233 & 10.388 \\
\cline { 2 - 4 } & PSNR & $\mathbf{1 4 . 4 0 2 8}$ & 15.9096 \\
$\begin{array}{c}\text { PSNR, SNR AND } \\
\text { CORRELATION } \\
\text { COEFFICIENT WHEN } \\
\text { NOISE VARIANCE IS.0.040 }\end{array}$ & SNR & $\mathbf{9 . 6 1 1 6}$ & 10.386 \\
\cline { 2 - 4 } & CC & $\mathbf{0 . 0 7 1 6}$ & $\mathbf{0 . 0 9 3 7}$ \\
\hline $\begin{array}{c}\text { PSNR, SNR AND } \\
\text { CORRELATION } \\
\text { COEFFICIENT WHEN } \\
\text { NOISE VARIANCE IS 0.060 }\end{array}$ & PSNR & 14.3821 & 15.9062 \\
\cline { 2 - 4 } & SNR & 9.590 & 10.38 \\
\cline { 2 - 4 } & CC & 0.0707 & 0.0945 \\
\hline
\end{tabular}


Table3: Impact of salt and pepper noise on watermark and recover watermark

\begin{tabular}{|c|c|c|c|}
\hline \multicolumn{2}{|l|}{ COMPARISION } & \multirow{2}{*}{$\begin{array}{l}\text { W1 \& } \\
\text { RW1 } \\
25.5776\end{array}$} & \multirow{2}{*}{$\begin{array}{l}\text { W2 \& } \\
\text { RW2 } \\
27.1281\end{array}$} \\
\hline \multirow{3}{*}{$\begin{array}{c}\text { PSNR, SNR AND } \\
\text { CORRELATION } \\
\text { COEFFICIENT WHEN } \\
\text { NOISE VARIANCE IS } \\
\mathbf{0 . 0 2 0}\end{array}$} & PSNR & & \\
\hline & SNR & 22.786 & 22.291 \\
\hline & $\mathrm{CC}$ & 0.0720 & 0.6417 \\
\hline \multirow{3}{*}{$\begin{array}{c}\text { PSNR, SNR AND } \\
\text { CORRELATION } \\
\text { COEFFICIENT WHEN } \\
\text { NOISE VARIANCE } \\
\text { IS.0.040 }\end{array}$} & PSNR & 26.074 & 27.120 \\
\hline & SNR & 21.283 & 22.281 \\
\hline & $\mathrm{CC}$ & 0.0669 & 0.6406 \\
\hline \multirow{3}{*}{$\begin{array}{c}\text { PSNR, SNR AND } \\
\text { CORRELATION } \\
\text { COEFFICIENT WHEN } \\
\text { NOISE VARIANCE IS } \\
0.060\end{array}$} & PSNR & 24.992 & 27.088 \\
\hline & SNR & 20.201 & 22.250 \\
\hline & $\mathbf{C C}$ & 0.0612 & 0.6359 \\
\hline
\end{tabular}

\section{OUT PUT GRAPH}

InGraph1[A],Graph1[B],Graph[C]andGraph2[A],Graph2[B], Green Line show Salt \& Pepper Noise, Red Line Show Speckle noise and Black Line represent impact of Gaussian Noise.

Graph1[A],Graph1[B],Graph[C] Represent PSNR,SNR,CC Comparison between watermark1 and recover watermark1.andGraph2[A],Graph2[B],represent PSNR , SNR comparison between watermark 2 and recover watermark2

Table4: Impact of Gaussian noise on watermark and recover watermark.

\begin{tabular}{|c|c|c|c|}
\hline \multicolumn{2}{|c|}{ COMPARISION } & W1 \& RW1 & W2 \& RW2 \\
\hline \multirow{2}{*}{$\begin{array}{c}\text { PSNR, SNR AND } \\
\text { CORRELATION } \\
\text { COEFFICIENT } \\
\text { WHEN NOISE } \\
\text { VARIANCE IS 0.020 }\end{array}$} & PSNR & 12.1535 & 15.9096 \\
\cline { 2 - 4 } & & & \\
\cline { 2 - 4 } & CNR & 7.3623 & 10.3890 \\
\hline $\begin{array}{c}\text { PSNR, SNR AND } \\
\text { CORRELATION } \\
\text { COEFFICIENT } \\
\text { WHEN NOISE } \\
\text { VARIANCE IS.0.040 }\end{array}$ & PSNR & $\mathbf{0 . 0 4 3 2}$ & 0.0941 \\
\cline { 2 - 4 } & CNR & 7.3514 & 15.9071 \\
\hline $\begin{array}{c}\text { PSNR, SNR AND } \\
\text { CORRELATION } \\
\text { COEFFICIENT } \\
\text { WHEN NOISE } \\
\text { VARIANCE IS 0.060 }\end{array}$ & PSNR & 12.561 & 15.9058 \\
\cline { 2 - 4 } & & & \\
\cline { 2 - 4 } & SNR & 7.7707 & 10.3865 \\
\hline
\end{tabular}

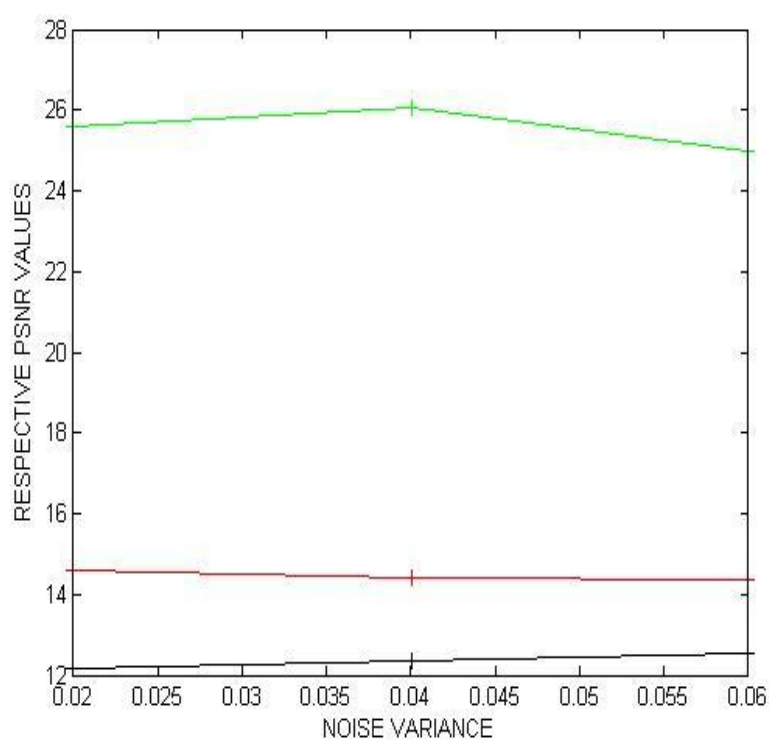

Graph 1[A]

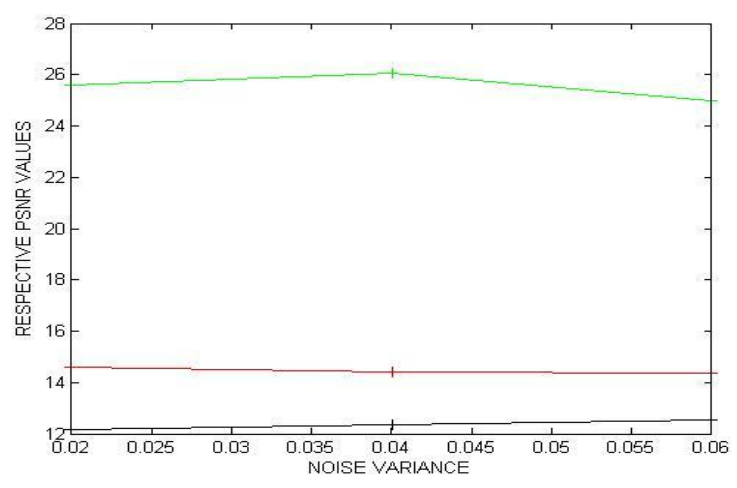

Graph 1[B]

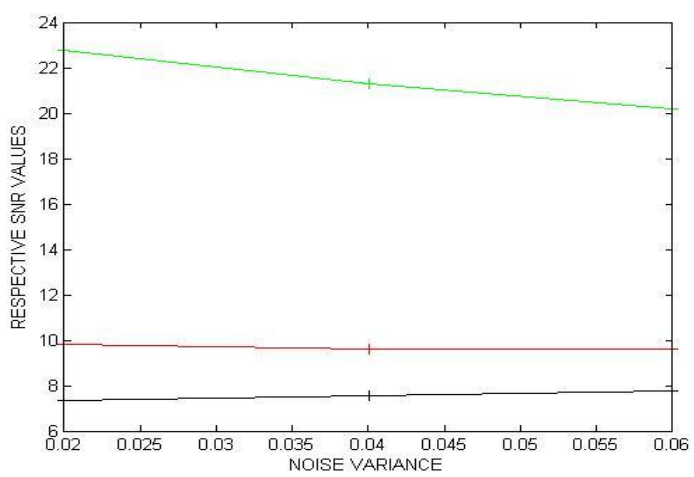

Graph 1[C] 


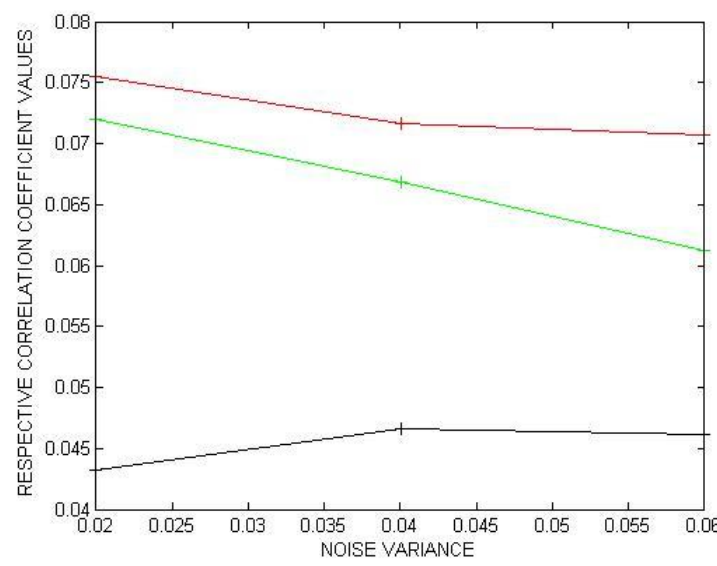

Graph 2[A]

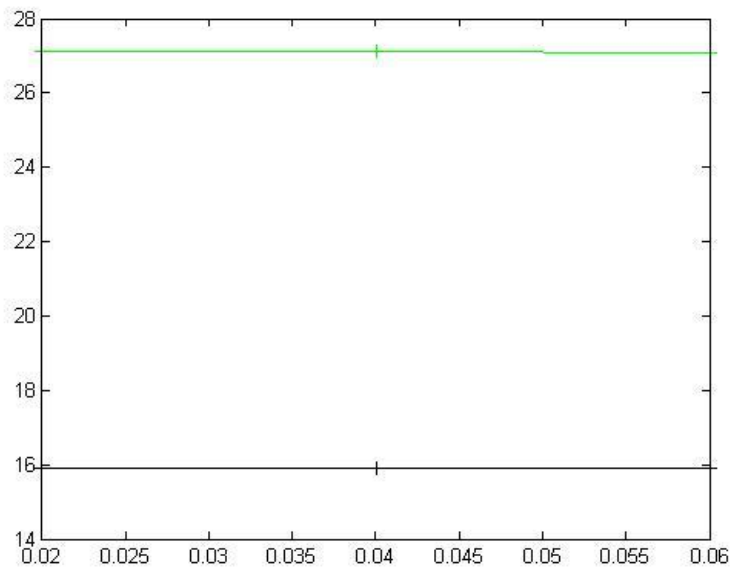

Graph 2[B]

\section{CONCLUSIONS}

In this paper author combine edge detection with watermarking for the purpose of highly authentication of original image. And here author also combine LSB and DCT watermarking for the purpose of providing highly security of image. But in case of noise attack on watermarked image what the effect of watermark image and recover watermark image also explain. In this paper Author check quality of image in the form of PSNR, SNR, and Correlation Coefficient when different- 2 type of noise with different- 2 variance attack on watermarked image.

\section{FUTURE SCOPE}

Now a day's image watermarking is the largest area of research. In this paper author concentrates only multi-level image based data security using LSB and DCT watermarking and it also show the effect of noise on image. In future I would like to extend this research in such a way, to develop an application that remove the effect of noise on cover object and recover watermark image.

\section{REFERENCES}

[1.] C.Cachin "An Information-Theoretic Model for Steganography", Proceedings of 2nd Workshop on Information Hiding, MIT Lab. for Computer Science, May 1998.

[2.] R.Gonzalez and Woods, "Digital Image Processing", 1998.

[3.] Q. Zhang, Z.Ji, W. Zhu, J. Lu and Y.-Q. Zhang, "Joint power control and source-channel coding for video communication over wireless," IEEE VTC'01, New Jersey, October 2001

[4.] Mandhani , N.K. (2004), "Watermarking Using Decimal Sequences", Master thesis submitted to the Graduate Faculty of the Louisiana State University, India

[5.] SarajuP.Mohanty, K.R. Ramakrishnan, Mohan Kankanhalli" A Dual Watermarking Technique for Images"

[6.] Baisa L. Gunjal , R.R. Manthalkar" an overview of transform domain robust digital image watermarking algorithms" Journal of Emerging Trends in Computing and Information Sciences, ISSN 2079-8407 Volume 2 No. 1

[7.] Abdullah Bamatraf, Rosziati Ibrahim and Mohd. NajibMohd. Salleh" A New Digital Watermarking Algorithm Using Combination of Least Significant Bit (LSB) and Inverse Bit" journal of computing, volume 3, issue 4, april 2011, issn 2151-9617

[8.] Tribhuwan Kumar Tewari, VikasSaxena" An Improved and Robust DCT based Digital Image Watermarking Scheme" International Journal of Computer Applications (0975 - 8887) Volume 3- No.1, June 2010.

[9.] SOBEL, I., An Isotropic $3 \times 3$ Gradient Operator, Machine Vision for Three -Dimensional Scenes, Freeman, H., Academic Pres, NY, 376-379, 1990.

[10.]SOBEL, I., Camera Models and Perception, Ph.D. thesis, Stanford University, Stanford, CA, 1970.

[11.]PREWITT, J., Object Enhancemet And Extraction, Picture Processing and Psychopictorics( B. Lipkin and A.Rosenfeld, Ed.), NY, Academic Pres, 1970.

[12.]ROBERTS, L. G., Machine Perception of ThreeDimensional Solids, in optical and Electro-Optical Information Processing ( J. Tippett, Ed.), 159-197, MIT Pres, 1965.

[13.]CANNY, J., A Computational Approach to Edge Detection, IEEE Transactions on Pattern Analysis and Machine Intelligence, 8, 679-700, 1986.

[14.]ZIOU, D. And TABBONE, S., Edge Detection Techniques - An Overview, Technical Report, No. 195, Dept. Math \&Informatique, University de Sherbrooke, 1997.

[15.]SHIGERU, A., Consistent Gradient Operators, IEEE Transactions on Pattern Analysis and Machine Intelligence, 22 (3), 2000. 